\title{
CC-MUSIC: An Optimization Estimator for Mutual Coupling Correction of L-Shaped Nonuniform Array with Single Snapshot
}

\author{
Yuguan Hou, ${ }^{1}$ Tongyu Zhang, ${ }^{2}$ and Shaochuan $\mathrm{Wu}^{1}$ \\ ${ }^{1}$ School of Electronics and Information Engineering, Harbin Institute of Technology, 92 West Dazhi Street, \\ Nangang, Harbin 150001, China \\ ${ }^{2}$ Institute of Engineering Mechanics, China Earthquake Administration, No. 29 Xuefu Road, Nangang, Harbin 150080, China
}

Correspondence should be addressed to Shaochuan Wu; scwu@hit.edu.cn

Received 2 September 2014; Revised 6 February 2015; Accepted 6 February 2015

Academic Editor: Massimo Scalia

Copyright (C) 2015 Yuguan Hou et al. This is an open access article distributed under the Creative Commons Attribution License, which permits unrestricted use, distribution, and reproduction in any medium, provided the original work is properly cited.

\begin{abstract}
For the case of the single snapshot, the integrated SNR gain could not be obtained without the multiple snapshots, which degrades the mutual coupling correction performance under the lower SNR case. In this paper, a Convex Chain MUSIC (CC-MUSIC) algorithm is proposed for the mutual coupling correction of the L-shaped nonuniform array with single snapshot. It is an online self-calibration algorithm and does not require the prior knowledge of the correction matrix initialization and the calibration source with the known position. An optimization for the approximation between the no mutual coupling covariance matrix without the interpolated transformation and the covariance matrix with the mutual coupling and the interpolated transformation is derived. A global optimization problem is formed for the mutual coupling correction and the spatial spectrum estimation. Furthermore, the nonconvex optimization problem of this global optimization is transformed as a chain of the convex optimization, which is basically an alternating optimization routine. The simulation results demonstrate the effectiveness of the proposed method, which improve the resolution ability and the estimation accuracy of the multisources with the single snapshot.
\end{abstract}

\section{Introduction}

The L-shaped linear array generally consists of two mutually perpendicular configuration uniform linear arrays, which could be horizontal or vertical. It can be used to estimate the target signal's two-dimensional information of the elevation angle and the azimuth angle. In addition, its structure is simpler than the planar linear array. In practical applications, the antenna performance of the L-shaped linear array is influenced by the mutual coupling between two array elements [1]. Ignoring the impact of the mutual coupling, the direction of arrival (DOA) estimation algorithm makes a serious degradation [2]. Reducing the impact of the mutual coupling of an antenna array, therefore, becomes an important task. The mutual coupling correction approach is a research hotspot in recent years. In the past two decades, many array calibration algorithms have been proposed for the mutual coupling problem. In [2], the authors pointed out that the mutual coupling reduced the eigenstructure decomposition algorithm performance, and a method of compensation and correction was introduced. In [3], a maximum likelihood algorithm for compensating the mutual coupling of the array element gain and phase has been proposed. This scheme considered all the obstacles without the calibration correction; however, a set of calibration sources in known locations were required. In [4], Lin and Yang considered the uniform circular arrays and presented a blind calibration method for the mutual coupling between elements. In [5], a method that links the DOA estimation and correction of the uniform linear array was presented. This method employed the Toeplitz structure of the array covariance matrix to compensate the gain and phase of the array elements. In [6], the self-correction of the array's mutual coupling was achieved by setting the amount of the auxiliary array element. Furthermore, the problems caused by iteration were also avoided. In [7], an online mutual coupling compensation algorithm for the uniform and linear arrays was presented. It could simultaneously compensate for the mutual coupling and estimate the direction-of-arrivals of signals. An alternating minimization procedure based on the closed-form solutions was 
performed to estimate the mutual coupling matrix in the field of complex symmetric Toeplitz matrices. In [8], a method for the estimation of the direction of arrival in the presence of multipath propagation and the mutual coupling for a frequency hopping system was proposed. An iterative alternating minimization algorithm for finding the mutual coupling and the DOA parameters in an alternate manner was formulated by using the pilot symbols and assuming perfect time-frequency synchronization for a linear array. In [9], a new mutual coupling compensation method based on the minimum norm solution to an underdetermined system of equations was introduced. The formulation was proved to be independent of the type of the antenna element and provide good results in situations where signal strengths vary considerably. The analysis of mutual coupling was applied in the context of a code division multiple access communication system. In [10], a computationally efficient algorithm for the direction of arrival estimation of uncorrelated sources and for self-calibration of mutual coupling between the sensors consisting of several uniformly spaced subarrays was proposed. In [11], a simple method was presented for the estimation and the compensation of mutual coupling in antenna systems of arbitrary geometries, including antenna arrays in the vicinity of scatters. The method included both theoretical and experimental schemes, whereas it did not resort to assumptions that often encountered in the previous mutual coupling estimation approaches. In [12], a decoupled method for $2 \mathrm{D}$ direction of arrival estimation in the presence of the elevation-dependent mutual coupling was proposed for the compact uniform circular arrays (UCAs) based on the rank reduction theory. In [13], a very simple but effective MUSIC DOA estimation algorithm was introduced by decoupling the antenna mutual coupling in the coupled noise component with the assumption that the uncoupled noise power can be determined a priori and can be removed from the array received power. In [14], the mutual-coupling problems in transmitting and receiving antenna arrays were revisited. The differences between the mutual coupling and mutual impedances for transmitting and receiving antenna arrays are explained. In [15], provided the angularly-independent mutual coupling was treated as angularly-dependent complex array gains, the middle subarray was found to have the same complex array gains. Consequently, a way for parameterizing the steering vector was proposed and the corresponding method for joint estimation of DOAs and mutual coupling matrix using the whole array data was derived based on subspace principle. In [16], the system identification method was applied to the wideband mutual coupling compensation of the receiving arrays. Using the receiving mutual impedances of an antenna array which was calculated at different frequencies, a multiport compensation network was identified for the wideband mutual coupling compensation.

The above-mentioned methods for the mutual coupling correction are based on the case of the multiple snapshots. For the case of the single snapshot, the integrated SNR gain could not be obtained without the multiple snapshots, and the performance of those methods degrades significantly. Therefore, it is a challengeable work to calibrate the mutual coupling in the case of the single snapshot with the lower SNR level. Moreover, for the correlated signals and the nonuniform array geometry cases, an additional optimization for the interpolated matrix is required. The main contribution of the paper is a joint optimization method for the online calibration of the mutual coupling in the cases of the single snapshot, the correlated signals, and the L-shaped nonuniform array geometry. A Convex Chain MUSIC (CC-MUSIC) for the Lshaped nonuniform array with single snapshot is proposed. It uses a novel iterative approach to improve the self-calibration algorithm for the mutual coupling correction. Neither the prior knowledge of the correction matrix initialization nor the calibration source with the known position is required for the proposed algorithm. The virtual array interpolation method is employed to transform the L-shaped linear nonuniform array to the virtual L-shaped linear uniform array for the spatial smoothing technique, which achieves a good DOA resolution and estimation for the coherent source signals. Subsequently an optimum solution for the approximation between the no mutual coupling covariance matrix without the interpolated transformation and the covariance matrix with the coupling and the interpolated transformation is derived. Moreover, a global optimization problem is formed for the mutual coupling correction and the spatial spectrum estimation. To avoid solving the nonconvex optimization problem, a chain of the convex optimization is adopted.

\section{Signal Model of Horizontal L-Shaped Nonuniform Linear Array for Coherent Sources}

Without loss of generality, we have the following assumptions to simplify the problem: the number of sources is smaller than that of the array elements, thus assuming that the number of the rows of the array bearing matrix is greater than that of the columns. The target signals are the far-field narrowband signals. For the horizontal L-shaped linear array [1], we assume that the reference element is at the origin of the antenna array coordinate axis as shown in Figure 1. The horizontal L-shaped nonuniform linear array is formed based on the horizontal L-shaped uniform linear array and some elements of which are failed or do not exist due to the restriction of the location place.

Denote the azimuth and elevation angles as $\theta$ and $\varphi$, respectively. Let the minimum unit of the adjacent distance between the elements be $d, d \leq \lambda / 2$, where $\lambda$ is the radar wavelength. Therefore, the adjacent distances between the elements are $1 \times d, 2 \times d, 3 \times d$, and so on. Let the number of the array elements on the $x$-axis be $N_{1}$, and let the number of the array elements on the $y$-axis be $N_{2}$. Then the total number of the array elements is $N=N_{1}+N_{2}$. Let the array element coordinate be $\left(x_{i}, y_{i}\right)\left(i=1,2, \ldots, N_{1}+N_{2}\right)$; then the phase difference between the $i$ th element and the reference element is

$$
\beta_{i}=\frac{2 \pi\left(\bar{x}_{i} \cos \theta \cos \varphi+\bar{y}_{i} \sin \theta \cos \varphi\right)}{\lambda},
$$




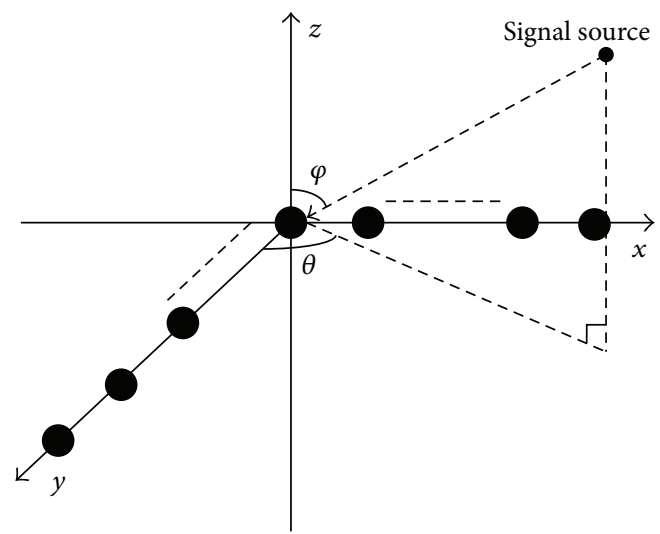

FIGURE 1: Horizontal L-shaped nonuniform linear array.

where

$$
\begin{aligned}
& \bar{x}_{i}= \begin{cases}\operatorname{dis}_{i 1} & 1 \leq i \leq N_{1} \\
0 & N_{1}+1 \leq i \leq N_{1}+N_{2},\end{cases} \\
& \bar{y}_{i}= \begin{cases}0 & 1 \leq i \leq N_{1} \\
\operatorname{dis}_{i 1} & N_{1}+1 \leq i \leq N_{1}+N_{2},\end{cases}
\end{aligned}
$$

where dis $_{i 1}$ indicates the distance between the $i$ th array element and the 1th element (i.e., the reference element).

We assume that the $K$ far-field narrowband signals enter into the antenna array. The $k$ th signal of the reference element $s_{k}(\hat{i})$ with the single snapshot $\hat{i}$ could be denoted as

$$
s_{k}(\hat{i})=u_{k}(\hat{i}) e^{j \phi(\hat{i})},
$$

where $u_{k}(\widehat{i})$ denotes the complex envelope of the signal and $\phi(\hat{i})$ represents the phase of the received signal. The signal on the $n$th array element $y_{n}(\hat{i})$ is denoted as

$$
y_{n}(\hat{i})=\sum_{k=1}^{K} s_{k}(\hat{i}) e^{-j 2 \pi f \tau_{n k}}+\eta_{n}(\hat{i}), \quad n=0,1, \ldots, N-1,
$$

where $f$ is the radar working frequency, $\tau_{n k}$ is the delay of the $k$ th signal reaching the $n$th array element, and $\eta_{n}(\hat{i})$ is the additive Gaussian colored noise at the $\hat{i}$ snapshot of the $n$th array element, which is correlated with other array elements.

Without the mutual coupling error, the steering vector of the L-shaped non-uniform linear array could be written as

$$
\mathbf{a}(\theta, \varphi)=\left[\mathbf{a}_{(x)}^{T}(\theta, \varphi), \mathbf{a}_{(y)}^{T}(\theta, \varphi)\right]^{T}
$$

where $(\cdot)^{T}$ represents the transpose of matrix/vector $(\cdot)$ and $\mathbf{a}_{(x)}(\theta, \varphi)$ and $\mathbf{a}_{(y)}(\theta, \varphi)$ are the steering vectors of $x$-axis and $y$-axis, respectively. We have

$$
\begin{aligned}
& \mathbf{a}_{(x)}(\theta, \varphi)=\left[1, e^{-j \beta_{2}}, \ldots, e^{-j \beta_{N_{1}}}\right]^{T}, \\
& \mathbf{a}_{(y)}(\theta, \varphi)=\left[1, e^{-j \beta_{N_{1}+2}}, \ldots, e^{-j \beta_{N_{1}+N_{2}}}\right]^{T} .
\end{aligned}
$$

In order to facilitate the analysis on the mutual coupling case, we suppose $N_{1}=N_{2}=N$. The total number of array elements is $2 N$. Denoting $\mathbf{Y}(\hat{i})$ as the vector of the single snapshot of the array output data, we have

$$
\mathbf{Y}(\hat{i})=\mathbf{M A}(\theta, \varphi) \mathbf{S}(\widehat{i})+\boldsymbol{\eta}(\hat{i})
$$

where

$$
\begin{gathered}
\mathbf{A}=\left(\begin{array}{cccc}
1 & 1 & \ldots & 1 \\
e^{-j \beta_{21}} & e^{-j \beta_{22}} & \ldots & e^{-j \beta_{2 K}} \\
\vdots & \vdots & \ddots & \vdots \\
e^{-j \beta_{\left(N_{1}+N_{2}\right) 1}} & e^{-j \beta_{\left(N_{1}+N_{2}\right) 2}} & \cdots & e^{-j \beta_{\left(N_{1}+N_{2}\right) K}}
\end{array}\right), \\
\mathbf{S}(\hat{i})=\left[s_{1}(\hat{i}), s_{2}(\widehat{i}), \ldots, s_{K}(\hat{i})\right]^{T}, \\
\boldsymbol{\eta}(\hat{i})=\left[\eta_{0}(\hat{i}), \eta_{1}(\hat{i}), \ldots, \eta_{N-1}(\hat{i})\right]^{T},
\end{gathered}
$$

where $\beta_{i k}(i=1,2, \ldots, 2 N$ and $k=1,2, \ldots, K)$ is the phase difference between the $i$ th element and the reference element for the $k$ th signal source. $\mathbf{A}(\theta, \varphi)$ is the bearing matrix of the array. $\mathbf{S}(\hat{i})$ is the signal vector. $\boldsymbol{\eta}(\hat{i})$ is the noise vector. $\mathbf{M}$ is the $2 N \times 2 N$ dimensional mutual coupling matrix, which is defined as

$$
\mathbf{M}=\left[\begin{array}{ll}
\mathbf{D} & \widehat{\mathrm{D}} \\
\widehat{\mathrm{D}} & \mathrm{D}
\end{array}\right]
$$

For the L-shaped linear array which consists of two mutually perpendicular nonuniform linear arrays, $\mathbf{D}$ is the $N \times N$ dimension mutual coupling matrix within $x$-axis or $y$-axis linear array and $\widehat{\mathbf{D}}$ is the $N \times N$ dimension mutual coupling matrix between the two linear arrays.

The mutual coupling matrix $\mathbf{D}$, which has the symmetric structure, could be described by the vector $\bar{\rho}=$ $\left[\rho_{0 d}, \ldots, \rho_{\operatorname{dis}_{i j}}, \ldots, \rho_{p 1 d}\right]$, where $\rho_{\operatorname{dis}_{i j}}$ is the mutual coupling coefficient between the $i$ th array element and the $j$ th array element and dis $_{i j}$ is the distance. The elements of the matrix $\mathbf{D}$ are expressed as

$$
\begin{aligned}
& \mathbf{D}_{i j}=\rho_{\mathrm{dis}_{i j}}=\mathbf{D}_{j i}, \\
& \mathbf{D}_{i j}=0 \quad \operatorname{dis}_{i j}>p 1 \cdot d, \\
& \mathbf{D}_{i i}=1 \quad i=j,
\end{aligned}
$$

where $i=1,2, \ldots, N_{1}, j=1,2, \ldots, N_{1}$ and $\mathbf{D}_{i j}$ is the element in the $i$ th row and $j$ th column of the mutual coupling matrix D. When the array element spacing is greater than $p 1 \cdot d$, the mutual coupling coefficient between the antenna elements is approximately zero, where $p 1$ is the mutual coupling linear degree of freedom. Therefore, the mutual coupling matrix within the ULA could be represented by a unique 
characterization of the $p 1$-dimensional vector. Similarly, the symmetric matrix $\widehat{\mathbf{D}}$ could be described by

$$
\begin{gathered}
\widehat{\mathbf{D}}_{1 j}=\mathbf{D}_{1 j}, \quad \widehat{\mathbf{D}}_{i 1}=\mathbf{D}_{i 1}, \\
\widehat{\mathbf{D}}_{i j}=0 \quad \operatorname{dis}_{i j}>p 2 \cdot d, \\
\widehat{\mathbf{D}}_{i j}=\widehat{\mathbf{D}}_{j i},
\end{gathered}
$$

where $i=1,2, \ldots, N_{2}, j=1,2, \ldots, N_{2}$ and $\widehat{\mathbf{D}}_{i j}$ is the element in the $i$ th row and $j$-column, $p 2$ is the mutual coupling linear degree of freedom between the linear arrays (similar with $p 1) . \mathbf{D}_{1 j}$ is the mutual coupling coefficient between the 1st element and the other elements within the same linear array. $\widehat{\mathbf{D}}_{1 j}$ is the mutual coupling coefficient between the 1st element and the elements on the another linear array (the coupling coefficients between the two linear arrays). The matrix $\widehat{\mathbf{D}}$ could be divided into two separate parts. Some identical elements with those of $\mathbf{D}$ are included in the 1st part $\widehat{\mathbf{D}}^{(1)}$, and the elements of the 2nd part $\widehat{\mathbf{D}}^{(2)}$ are different from those of $\mathbf{D}$; that is, $\widehat{\mathbf{D}}=\widehat{\mathbf{D}}^{(1)}+\widehat{\mathbf{D}}^{(2)} \cdot \widehat{\mathbf{D}}^{(2)}$ could be described by the vector $\widehat{\boldsymbol{\rho}}^{(2)}=\left[\hat{\rho}_{0 d}, \ldots, \hat{\rho}_{\mathrm{di}_{i j}}, \ldots, \hat{\rho}_{p 2 d}\right]$, where $\widehat{\rho}_{\mathrm{dis}_{i j}}$ is the mutual coupling coefficient between the $i$ th array element and the $j$ th array element of the another linear array, and the upper triangle part of the symmetric matrix $\widehat{\mathbf{D}}^{(2)}(2: p 2+1,2$ : $p 2+1)$ is filled with the elements of the vector. Thus, the mutual coupling matrix $\mathbf{M}$ is composed of the parameters including $\rho_{0 d}, \ldots, \rho_{\mathrm{dis}_{i j}}, \ldots, \rho_{p 1 d}$ and $\widehat{\rho}_{0 d}, \ldots, \widehat{\rho}_{\mathrm{di}_{i j}}, \ldots, \widehat{\rho}_{p 2 d}$. And we have

$$
\mathbf{M a}(\theta, \varphi)=\left[\begin{array}{c}
\mathbf{D} \mathbf{a}_{(x)}(\theta, \varphi)+\widehat{\mathbf{D}} \mathbf{a}_{(y)}(\theta, \varphi) \\
\widehat{\mathbf{D}} \mathbf{a}_{(x)}(\theta, \varphi)+\mathbf{D} \mathbf{a}_{(y)}(\theta, \varphi)
\end{array}\right] .
$$

The array covariance matrix of the single snapshot could be expressed as

$$
\mathbf{R}=\mathbf{Y}(\widehat{i}) \mathbf{Y}^{H}(\widehat{i})=\mathbf{M A R}_{S} \mathbf{A}^{H} \mathbf{M}^{H}+\mathbf{\Gamma},
$$

where $\Gamma$ indicates the covariance matrix of the noise.

In the case of the single snapshot, the multisources need to be treated as the coherent sources for a better resolution. Therefore, the virtual array technique $[17,18]$ of the nonuniform linear array is used for the resolution of the coherent sources signals. To design the interpolated array, the field of view of the array is divided into some sectors. For example, two sectors are defined by the interval $\left[\theta^{(1)}, \theta^{(2)}\right]$ and $\left[\varphi^{(1)}, \varphi^{(2)}\right]$. Denote the sets of angles $\boldsymbol{\theta}^{s}$ and $\varphi^{s}$ as

$$
\begin{aligned}
\boldsymbol{\theta}^{s} & =\left[\theta^{(1)}, \theta^{(1)}+\Delta \theta, \theta^{(1)}+2 \Delta \theta, \ldots, \theta^{(2)}\right], \\
\boldsymbol{\varphi}^{s} & =\left[\varphi^{(1)}, \varphi^{(1)}+\Delta \varphi, \varphi^{(1)}+2 \Delta \varphi, \ldots, \varphi^{(2)}\right],
\end{aligned}
$$

where $\Delta \theta$ and $\Delta \varphi$ are the azimuth and elevation angle intervals of the sectors, respectively. These angles are used only in the design of the interpolation matrices. The steering vectors associated with the sets $\boldsymbol{\theta}^{s}$ and $\boldsymbol{\varphi}^{s}$ for the given array are computed and arranged in the matrices form as follows:

$$
\begin{aligned}
\mathbf{A}_{\theta} & =\left[\mathbf{a}_{(x)}\left(\theta^{(1)}\right), \ldots, \mathbf{a}_{(x)}\left(\theta^{(2)}\right)\right], \\
\mathbf{A}_{\varphi} & =\left[\mathbf{a}_{(y)}\left(\varphi^{(1)}\right), \ldots, \mathbf{a}_{(y)}\left(\varphi^{(2)}\right)\right] .
\end{aligned}
$$

In other words, $\mathbf{A}_{\theta}$ and $\mathbf{A}_{\varphi}$ are the sections of the array manifold of the real array. Denote $\overline{\mathbf{A}}_{\theta}$ and $\overline{\mathbf{A}}_{\varphi}$ as the sections of the interpolated virtual array manifold computed for the sets of angles $\boldsymbol{\theta}^{s}$ and $\boldsymbol{\varphi}^{s}$ :

$$
\begin{aligned}
\overline{\mathbf{A}}_{\theta} & =\left[\overline{\mathbf{a}}_{(x)}\left(\theta^{(1)}\right), \ldots, \overline{\mathbf{a}}_{(x)}\left(\theta^{(2)}\right)\right], \\
\overline{\mathbf{A}}_{\varphi} & =\left[\overline{\mathbf{a}}_{(y)}\left(\varphi^{(1)}\right), \ldots, \overline{\mathbf{a}}_{(y)}\left(\varphi^{(2)}\right)\right],
\end{aligned}
$$

where $\overline{\mathbf{a}}_{(x)}\left(\theta^{(1)}\right)$ and $\overline{\mathbf{a}}_{(y)}\left(\varphi^{(1)}\right)$ are the responses of the interpolated virtual array of $x$-axis and $y$-axis. The interpolated virtual array is an L-shaped uniform linear array, which has the same number of elements as the real array. However, the adjacent distances of the elements are different. Assuming that there exists the interpolated transformation matrices $\mathbf{B}_{\theta}$ and $\mathbf{B}_{\varphi}$, we have

$$
\begin{aligned}
\mathbf{B}_{\theta} \mathbf{A}_{\theta} & =\overline{\mathbf{A}}_{\theta}, \\
\mathbf{B}_{\varphi} \mathbf{A}_{\varphi} & =\overline{\mathbf{A}}_{\varphi} .
\end{aligned}
$$

In fact, the interpolations are not exact; therefore, the equations above are not really hold. The "best" interpolation matrices are those giving the best fit between the interpolated response $\mathbf{B}_{\theta} \mathbf{A}_{\theta}$ and the desired response $\overline{\mathbf{A}}_{\theta}$ and between the interpolated response $\mathbf{B}_{\varphi} \mathbf{A}_{\varphi}$ and the desired response $\overline{\mathbf{A}}_{\varphi}$. Denote the L-shaped interpolated transformation matrix as

$$
\mathbf{B}=\left[\begin{array}{cc}
\mathbf{B}_{\theta} & \mathbf{0} \\
\mathbf{0} & \mathbf{B}_{\varphi}
\end{array}\right] .
$$

Given the interpolation matrix we can calculate the array data vector $\overline{\mathbf{Y}}(\hat{i})$ of the virtual array

$$
\overline{\mathbf{Y}}(\hat{i})=\mathbf{B Y}(\hat{i}) .
$$

The interpolation matrix $\mathbf{B}$ is equal to

$$
\mathbf{B}=\left[\begin{array}{cc}
\overline{\mathbf{A}}_{\theta} \mathbf{A}_{\theta}^{H}\left(\mathbf{A}_{\theta} \mathbf{A}_{\theta}^{H}\right)^{-1} & \mathbf{0} \\
\mathbf{0} & \overline{\mathbf{A}}_{\varphi} \mathbf{A}_{\varphi}^{H}\left(\mathbf{A}_{\varphi} \mathbf{A}_{\varphi}^{H}\right)^{-1}
\end{array}\right],
$$

where $(\cdot)^{H}$ represents the conjugate transpose of (.). As $\mathbf{A}_{\theta}$ and $\mathbf{A}_{\varphi}$ are known and the number of elements of the virtual array is equal to that of the real array, the matrix B is determined by the value of the adjacent distances of the elements of the virtual array. With the transformation as shown in (19), the L-shaped nonuniform linear array is transformed into the virtual L-shaped uniform linear array. Therefore, the forward-backward smoothing technique [19] could be employed for the resolution of the coherent sources. 
Dividing the virtual array into $M$ virtual subarrays within the $x$-axis and the $y$-axis, respectively, the number of the elements of the subarray is $N_{M}=N-M+1$. The data vector of the $m$ th $(m=1,2, \ldots, M)$ subarray within the $x$-axis and $y$-axis is denoted as

$$
\begin{aligned}
\overline{\mathbf{Y}}_{m}^{(x)}(\hat{i}) & =\left[\bar{y}_{m}^{(x)}(\hat{i}), \bar{y}_{m+1}^{(x)}(\hat{i}), \ldots, \bar{y}_{m+N_{M^{-1}}}^{(x)}(\hat{i})\right]^{T}, \\
\overline{\mathbf{Y}}_{m}^{(y)}(\hat{i}) & =\left[\bar{y}_{m}^{(y)}(\hat{i}), \bar{y}_{m+1}^{(y)}(\hat{i}), \ldots, \bar{y}_{m+N_{M^{-1}}}^{(y)}(\hat{i})\right]^{T},
\end{aligned}
$$

where $\bar{y}_{m}^{(x)}(\hat{i})(m=1,2, \ldots, M)$ is the $m$ th element of the data vector of the virtual array within the $x$-axis and $\bar{y}_{m}^{(y)}(\hat{i})(m=$ $1,2, \ldots, M)$ is the $m$ th element of the data vector of the virtual array within the $y$-axis. Thus, the covariance matrix of the $m$ th $(m=1,2, \ldots, M)$ subarray with the single snapshot is expressed as

$$
\begin{aligned}
\overline{\mathbf{R}}_{m}^{(x)} & =\overline{\mathbf{Y}}_{m}^{(x)}(\hat{i}) \overline{\mathbf{Y}}_{m}^{(x)^{H}}(\hat{i}), \\
\overline{\mathbf{R}}_{m}^{(y)} & =\overline{\mathbf{Y}}_{m}^{(y)}(\hat{i}) \overline{\mathbf{Y}}_{m}^{(y)^{H}}(\hat{i}) .
\end{aligned}
$$

The forward-backward smoothing covariance matrix is denoted as

$$
\begin{aligned}
& \overline{\mathbf{R}}^{(x)}=\frac{1}{2 M} \sum_{m=1}^{M}\left(\overline{\mathbf{R}}_{m}^{(x)}+\mathbf{J}\left(\overline{\mathbf{R}}_{m}^{(x)}\right)^{*} \mathbf{J}\right), \\
& \overline{\mathbf{R}}^{(y)}=\frac{1}{2 M} \sum_{m=1}^{M}\left(\overline{\mathbf{R}}_{m}^{(y)}+\mathbf{J}\left(\overline{\mathbf{R}}_{m}^{(y)}\right)^{*} \mathbf{J}\right),
\end{aligned}
$$

where $(\cdot)^{*}$ denotes conjugation and $\mathbf{J}$ is $M \times M$ exchange matrix with the antidiagonal elements being 1 and the other elements being 0 . The characteristics of the covariance matrix could be obtained through the eigendecomposition. With the noise subspace matrixes $\overline{\mathbf{V}}_{n}^{(x)}$ and $\overline{\mathbf{V}}_{n}^{(y)}$, the MUSIC [20] spatial spectrum estimation equations of the $x$-axis and the $y$-axis are shown in the following equation:

$$
\begin{gathered}
\left.P_{\text {MUSIC }}^{(x)}(\theta, \varphi)\right|_{\varphi=\varphi_{0}}=\left.\frac{1}{\left\|\overline{\mathbf{V}}_{n}^{(x) H_{\overline{\mathbf{a}}}(x)}(\theta, \varphi)\right\|^{2}}\right|_{\varphi=\varphi_{0}}, \\
\left.P_{\text {MUSIC }}^{(y)}(\theta, \varphi)\right|_{\theta=\theta_{0}}=\left.\frac{1}{\left\|\overline{\mathbf{V}}_{n}^{(y) H} \overline{\mathbf{a}}_{(y)}(\theta, \varphi)\right\|^{2}}\right|_{\theta=\theta_{0}},
\end{gathered}
$$

where $\varphi_{0}$ is a given elevation angle and $\theta_{0}$ is a given azimuth angle.

\section{A Convex Chain Approach}

For the covariance matrix of the $m$ th $(m=1,2, \ldots, M)$ subarray of the $x$-axis or the $y$-axis, we define the no mutual coupling covariance matrix without the interpolated transformation as $\mathbf{R}_{1}$. The covariance matrix with the coupling and the interpolated transformation as $\overline{\mathbf{R}}_{2}$, and its approximation is denoted as

$$
\overline{\mathbf{R}}_{2}=\mathbf{B M R}_{1} \mathbf{M}^{\mathrm{H}} \mathbf{B}^{\mathbf{H}},
$$

where $\overline{\mathbf{R}}_{2}=\overline{\mathbf{R}}_{m}^{(x)}$ or $\overline{\mathbf{R}}_{2}=\overline{\mathbf{R}}_{m}^{(y)}, m=1,2, \ldots, M$. The solution of $\overline{\mathbf{R}}_{1}$ could be obtained by solving the following problem:

$$
\left(\mathbf{R}_{1}\right)_{\text {opt }}=\arg \min \left\|\overline{\mathbf{R}}_{2}-\mathbf{B} \mathbf{M} \mathbf{R}_{1} \mathbf{M}^{\mathrm{H}} \mathbf{B}^{\mathrm{H}}\right\|_{F}^{2} .
$$

In the single snapshot case and the SNR is not high enough. The noise component could not be ignored and the noise covariance matrix could not be expressed with the identical matrix of (26). Equation (26) is rewritten as

$$
\left(\mathbf{R}_{1}\right)_{\text {opt }}=\arg \min \left\|\overline{\mathbf{R}}_{2}-\mathbf{B} \mathbf{M B} B^{-1} \mathbf{B R}_{1} \mathbf{B}^{\mathrm{H}} \mathbf{B}^{-\mathbf{H}} \mathbf{M}^{\mathrm{H}} \mathbf{B}^{\mathbf{H}}\right\|_{F}^{2} .
$$

In order to alleviate the influence of the noise component, the Toeplitz approximation is introduced for the $\mathbf{B R}_{1} \mathbf{B}^{\mathbf{H}}$ which is transformed into the virtual uniform linear array. Therefore, we denote the Toeplitz matrix as

$$
\overline{\mathbf{R}}_{1}=\mathbf{B R}_{1} \mathbf{B}^{\mathbf{H}} .
$$

Equation (27) is, therefore, rewritten as

$$
\left(\mathbf{R}_{1}\right)_{\text {opt }}=\arg \min \left\|\overline{\mathbf{R}}_{2}-\mathbf{B M B}^{-1} \overline{\mathbf{R}}_{1} \mathbf{B}^{-\mathbf{H}} \mathbf{M}^{\mathrm{H}} \mathbf{B}^{\mathbf{H}}\right\|_{F}^{2} .
$$

The correlation matrix $\overline{\mathbf{R}}_{1}$ is composed of $\mathbf{r}_{1}$, and $\mathbf{r}_{1}$ is obtained from the matrix $\overline{\mathbf{R}}_{1}$. In order to emphasize the characteristic parameters of $\overline{\mathbf{R}}_{1}$, it is denoted as

$\mathbf{r}_{1}$

$$
\triangleq\left[\overline{\mathbf{R}}_{1}\left(N_{N_{M}}, 1\right), \ldots, \overline{\mathbf{R}}_{1}(2,1), \overline{\mathbf{R}}_{1}(1,1), \ldots, \overline{\mathbf{R}}_{1}\left(1, N_{N_{M}}\right)\right]^{T} .
$$

As $\overline{\mathbf{R}}_{1}$ is the complex conjugate Toeplitz matrix, it can be written as

$$
\begin{gathered}
\overline{\mathbf{R}}_{1} \mathbf{B}^{-\mathbf{H}}=\left[\mathbf{Q}_{h}\left(\left[\mathbf{B}^{-\mathbf{H}}\right]_{:, 1}\right) \mathbf{r}_{1}, \mathbf{Q}_{h}\left(\left[\mathbf{B}^{-\mathbf{H}}\right]_{:, 2}\right) \mathbf{r}_{1}, \ldots,\right. \\
\left.\mathbf{Q}_{h}\left(\left[\mathbf{B}^{-\mathbf{H}}\right]_{:, N_{M}}\right) \mathbf{r}_{1}\right],
\end{gathered}
$$

where $\left[\mathbf{B}^{-\mathbf{H}}\right]_{:, n}$ represents the $n$th column of the matrix $\mathbf{B}^{-\mathbf{H}}$, and for any column vector $\mathbf{x} \in \mathbb{C}^{N_{M}, 1}$ matrix $\mathbf{Q}_{h}(\mathbf{x}) \in$ $\mathbb{C}^{N_{M}, 2 N_{M^{-1}}}$ can be written as

$$
\mathbf{Q}_{h}(\mathbf{x})=\mathbf{W}_{h 1}(\mathbf{x})+\mathbf{W}_{h 2}(\mathbf{x}),
$$

where the matrixes $\mathbf{W}_{h 1}(\mathbf{x}) \in \mathbb{C}^{N_{M}, 2 N_{M}-1}$ and $\mathbf{W}_{h 2}(\mathbf{x}) \epsilon$ $\mathbb{C}^{N_{M}, 2 N_{M}-1}$ are defined as

$$
\begin{gathered}
{\left[\mathbf{W}_{h 1}(\mathbf{x})\right]_{p q}= \begin{cases}{[\mathbf{x}]_{p+q-N_{M}},} & \text { if } p+q \leq 2 N_{M}, q>N_{M} \\
0, & \text { else, }\end{cases} } \\
{\left[\mathbf{W}_{h 2}(\mathbf{x})\right]_{p q}= \begin{cases}{[\mathbf{x}]_{p+q-N_{M}},} & \text { if } p+q \geq N_{M}+1, \\
0, & q \leq N_{M}-1\end{cases} } \\
\hline 0 \text { else, }
\end{gathered}
$$


where $p=1,2, \ldots, N_{M}, q=1,2, \ldots, 2 N_{M}-1$. An equivalent and more compact form could be obtained by defining the following equation:

$$
\begin{aligned}
& \mathbf{Q}_{h}\left(\mathbf{B}^{-\mathbf{H}}\right) \\
& \triangleq\left[\mathbf{Q}_{h}\left(\left[\mathbf{B}^{-\mathbf{H}}\right]_{:, 1}\right), \mathbf{Q}_{h}\left(\left[\mathbf{B}^{-\mathbf{H}}\right]_{:, 2}\right), \ldots, \mathbf{Q}_{h}\left(\left[\mathbf{B}^{-\mathbf{H}}\right]_{:, N_{M}}\right)\right] \\
& \in \mathbb{C}^{N_{M}, N_{M}\left(2 N_{M}-1\right)} .
\end{aligned}
$$

Thus we can get

$$
\overline{\mathbf{R}}_{1} \mathbf{B}^{-\mathbf{H}}=\mathbf{Q}_{h}\left(\mathbf{B}^{-\mathbf{H}}\right)\left(\mathbf{I}_{N_{M}} \otimes \mathbf{r}_{1}\right) .
$$

Substituting (35) into (29), we have

$$
\begin{aligned}
& \left(\mathbf{R}_{1}\right)_{\mathrm{opt}} \\
& =\arg \min \left\|\overline{\mathbf{R}}_{2}-\mathbf{B M B}^{-1} \mathbf{Q}_{h}\left(\mathbf{B}^{-\mathbf{H}}\right)\left(\mathbf{I}_{N_{M}} \otimes \mathbf{r}_{1}\right) \mathbf{M}^{\mathbf{H}} \mathbf{B}^{\mathbf{H}}\right\|_{F}^{2} .
\end{aligned}
$$

The complex gradient of the function to be minimized is equal to zero; we have

$$
\nabla_{\mathbf{r}_{1}^{*}}\left\|\overline{\mathbf{R}}_{2}-\mathbf{B M B}^{-1} \mathbf{Q}_{h}\left(\mathbf{B}^{-\mathbf{H}}\right)\left(\mathbf{I}_{N_{M}} \otimes \mathbf{r}_{1}\right) \mathbf{M}^{\mathbf{H}} \mathbf{B}^{\mathbf{H}}\right\|_{F}^{2}=\mathbf{0}_{N_{M}},
$$

where $\mathbf{0}_{N_{M}}$ is an $N_{M} \times 1$ column vector made by all zeros. Firstly, the cost function is expanded as

$$
\begin{aligned}
& \left\|\overline{\mathbf{R}}_{2}-\mathbf{B M B}^{-1} \mathbf{Q}_{h}\left(\mathbf{B}^{-\mathbf{H}}\right)\left(\mathbf{I}_{N_{M}} \otimes \mathbf{r}_{1}\right) \mathbf{M}^{\mathbf{H}} \mathbf{B}^{\mathbf{H}}\right\|_{F}^{2} \\
& =\operatorname{tr}\left\{\left(\overline{\mathbf{R}}_{2}-\mathbf{B M B}^{-1} \mathbf{Q}_{h}\left(\mathbf{B}^{-\mathbf{H}}\right)\left(\mathbf{I}_{N_{M}} \otimes \mathbf{r}_{1}\right) \mathbf{M}^{\mathbf{H}} \mathbf{B}^{\mathbf{H}}\right)\right. \\
& \left.\cdot\left(\overline{\mathbf{R}}_{2}-\mathbf{B M B}^{-1} \mathbf{Q}_{h}\left(\mathbf{B}^{-\mathbf{H}}\right)\left(\mathbf{I}_{N_{M}} \otimes \mathbf{r}_{1}\right) \mathbf{M}^{\mathbf{H}} \mathbf{B}^{\mathbf{H}}\right)^{H}\right\} \\
& =\operatorname{tr}\left\{\overline{\mathbf{R}}_{2} \overline{\mathbf{R}}_{2}^{H}\right\} \\
& -\operatorname{tr}\left\{\mathbf{Q}_{h}^{H}\left(\mathbf{B}^{-\mathbf{H}}\right) \mathbf{B}^{-\mathbf{H}} \mathbf{M}^{\mathbf{H}} \mathbf{B}^{\mathbf{H}} \overline{\mathbf{R}}_{2} \mathbf{B M}\left(\mathbf{I}_{N_{M}} \otimes \mathbf{r}_{1}^{H}\right)\right\} \\
& -\operatorname{tr}\left\{\mathbf{M}^{\mathbf{H}} \mathbf{B}^{\mathbf{H}} \overline{\mathbf{R}}_{2}^{H} \mathbf{B M B}^{-1} \mathbf{Q}_{h}\left(\mathbf{B}^{-\mathbf{H}}\right)\left(\mathbf{I}_{N_{M}} \otimes \mathbf{r}_{1}\right)\right\} \\
& +\operatorname{tr}\left\{\mathbf{Q}_{h}^{H}\left(\mathbf{B}^{-\mathbf{H}}\right) \mathbf{B}^{-\mathbf{H}} \mathbf{M}^{\mathrm{H}} \mathbf{B}^{\mathrm{H}} \overline{\mathbf{R}}_{2} \mathbf{B} \mathbf{M} \mathbf{M}^{\mathrm{H}} \mathbf{B}^{\mathrm{H}} \mathbf{B} \mathbf{B B}^{-1}\right. \\
& \left.\cdot \mathbf{Q}_{h}\left(\mathbf{B}^{-\mathbf{H}}\right)\left(\mathbf{I}_{N_{M}} \otimes \mathbf{r}_{1}\right)\left(\mathbf{I}_{N_{M}} \otimes \mathbf{r}_{1}^{H}\right)\right\} .
\end{aligned}
$$

According to the theory of complex gradient, the quantity $\mathbf{r}_{1}$ should be treated as a constant, while the only variable term is $\mathbf{r}_{1}^{*}$. Therefore, it is derived that the complex gradients of the addends in (38) are given by

$$
\begin{gathered}
\nabla_{\mathbf{r}_{1}^{*}} \operatorname{tr}\left\{\overline{\mathbf{R}}_{2} \overline{\mathbf{R}}_{2}^{H}\right\}=\mathbf{0}_{N_{M}}, \\
\nabla_{\mathbf{r}_{1}^{*}} \operatorname{tr}\left\{\mathbf{Q}_{h}^{H}\left(\mathbf{B}^{-\mathbf{H}}\right) \mathbf{B}^{-\mathbf{H}} \mathbf{M}^{\mathbf{H}} \mathbf{B}^{\mathbf{H}} \overline{\mathbf{R}}_{2} \mathbf{B} \mathbf{M}\left(\mathbf{I}_{N_{M}} \otimes \mathbf{r}_{1}^{H}\right)\right\} \\
=\mathbf{J}_{N_{M}, 2 N_{M^{-1}}} \operatorname{vec}\left\{\mathbf{Q}_{h}^{H}\left(\mathbf{B}^{-\mathbf{H}}\right) \mathbf{B}^{-\mathbf{H}} \mathbf{M}^{\mathbf{H}} \mathbf{B}^{\mathrm{H}} \overline{\mathbf{R}}_{2} \mathbf{B} \mathbf{M}\right\}, \\
\nabla_{\mathbf{r}_{1}^{*}} \operatorname{tr}\left\{\mathbf{M}^{\mathbf{H}} \mathbf{B}^{\mathbf{H}} \overline{\mathbf{R}}_{2}^{H} \mathbf{B M B}^{-1} \mathbf{Q}_{h}\left(\mathbf{B}^{-\mathbf{H}}\right)\left(\mathbf{I}_{N_{M}} \otimes \mathbf{r}_{1}\right)\right\}=\mathbf{0}_{2 N_{M}-1} \\
\nabla_{\mathbf{r}_{1}^{*}} \operatorname{tr}\left\{\mathbf{Q}_{h}^{H}\left(\mathbf{B}^{-\mathbf{H}}\right) \mathbf{B}^{-\mathbf{H}} \mathbf{M}^{\mathbf{H}} \mathbf{B}^{\mathbf{H}} \overline{\mathbf{R}}_{2} \mathbf{B} \mathbf{M} \mathbf{M}^{\mathbf{H}} \mathbf{B}^{\mathbf{H}} \mathbf{B} \mathbf{M B} \mathbf{B}^{-1}\right. \\
\left.\cdot \mathbf{Q}_{h}\left(\mathbf{B}^{-\mathbf{H}}\right)\left(\mathbf{I}_{N_{M}} \otimes \mathbf{r}_{1}\right)\left(\mathbf{I}_{N_{M}} \otimes \mathbf{r}_{1}^{H}\right)\right\} \\
=\mathbf{J}_{N_{M}, 2 N_{M^{-1}}} \operatorname{vec}\left\{\mathbf{Q}_{h}^{H}\left(\mathbf{B}^{-\mathbf{H}}\right) \mathbf{B}^{-\mathbf{H}} \mathbf{M}^{\mathbf{H}} \mathbf{B}^{\mathbf{H}} \overline{\mathbf{R}}_{2} \mathbf{B}\right. \\
\left.\cdot \mathbf{M} \mathbf{M}^{\mathbf{H}} \mathbf{B}^{\mathbf{H}} \mathbf{B} \mathbf{M} \mathbf{B}^{-1} \mathbf{Q}_{h}\left(\mathbf{B}^{-\mathbf{H}}\right)\left(\mathbf{I}_{N_{M}} \otimes \mathbf{r}_{1}\right)\right\},
\end{gathered}
$$

where $\mathbf{J}_{N_{M}, 2 N_{M}-1} \in \mathbb{R}^{\left(2 N_{M}-1\right),\left(2 N_{M}-1\right) N_{M}^{2}}$ is a sparse selection matrix denoted as

$$
\begin{gathered}
\mathbf{J}_{N_{M}, 2 N_{M}-1} \triangleq\left[\begin{array}{c}
\operatorname{vec}^{T}\left\{\mathbf{I}_{N_{M}} \otimes \mathbf{e}_{1}\right\} \\
\operatorname{vec}^{T}\left\{\mathbf{I}_{N_{M}} \otimes \mathbf{e}_{2}\right\} \\
\vdots \\
\operatorname{vec}^{T}\left\{\mathbf{I}_{N_{M}} \otimes \mathbf{e}_{2 N_{M}-1}\right\}
\end{array}\right], \\
\mathbf{e}_{n} \triangleq[\underbrace{0, \ldots 0,1}_{n-1}, \underbrace{0, \ldots 0}_{2 N_{M}-1-n}]^{T} \in \mathbb{R}^{2 N_{M}-1,1} .
\end{gathered}
$$

For any complex $F G \times F$ matrix $\mathbf{C}$ and any complex $G \times 1$ vector $\mathbf{c}$, the following equation is true:

$$
\operatorname{tr}\left\{\mathbf{C}\left(\mathbf{I}_{F} \otimes \mathbf{c}^{T}\right)\right\}=\operatorname{vec}^{T}\left\{\mathbf{I}_{F} \otimes \mathbf{c}^{T}\right\} \operatorname{vec}\{\mathbf{C}\} .
$$

Therefore, (37) can be rewritten as

$$
\begin{gathered}
\nabla_{\mathbf{r}_{1}^{*}}\left\|\overline{\mathbf{R}}_{2}-\mathbf{B M B}^{-1} \mathbf{Q}_{h}\left(\mathbf{B}^{-\mathbf{H}}\right)\left(\mathbf{I}_{N_{M}} \otimes \mathbf{r}_{1}\right) \mathbf{M}^{\mathbf{H}} \mathbf{B}^{\mathbf{H}}\right\|_{F}^{2} \\
=\mathbf{J}_{N_{M}, 2 N_{M}-1} \operatorname{vec}\left\{\mathbf{Q}_{h}^{H}\left(\mathbf{B}^{-\mathbf{H}}\right) \mathbf{B}^{-\mathbf{H}} \mathbf{M}^{\mathbf{H}} \mathbf{B}^{\mathbf{H}} \overline{\mathbf{R}}_{2} \mathbf{B} \mathbf{M}\right\} \\
-\mathbf{J}_{N_{M}, 2 N_{M}-1} \operatorname{vec}\left\{\mathbf{Q}_{h}^{H}\left(\mathbf{B}^{-\mathbf{H}}\right) \mathbf{B}^{-\mathbf{H}} \mathbf{M}^{\mathbf{H}} \mathbf{B}^{\mathbf{H}} \overline{\mathbf{R}}_{2} \mathbf{B}\right. \\
\left.\cdot \mathbf{M M}^{\mathbf{H}} \mathbf{B}^{\mathbf{H}} \mathbf{B} \mathbf{M B} \mathbf{B}^{-1} \mathbf{Q}_{h}\left(\mathbf{B}^{-\mathbf{H}}\right)\left(\mathbf{I}_{N_{M}} \otimes \mathbf{r}_{1}\right)\right\} \\
=\mathbf{0}_{2 N_{M^{-1}}} .
\end{gathered}
$$

By exploiting the fact that

$$
\operatorname{vec}\{\mathbf{C D}\}=\left\{\mathbf{I}_{G} \otimes \mathbf{C}\right\} \operatorname{vec}\{\mathbf{D}\}
$$


for any complex $E \times F$ matrix $\mathbf{C}$ and any complex $F \times G$ matrix $\mathrm{D},(42)$ can be rewritten as

$$
\begin{gathered}
\mathbf{J}_{N_{M}, 2 N_{M}-1} \operatorname{vec}\left\{\mathbf { I } _ { N _ { M } } \otimes \left(\mathbf{Q}_{h}^{H}\left(\mathbf{B}^{-\mathbf{H}}\right) \mathbf{B}^{-\mathbf{H}} \mathbf{M}^{\mathbf{H}} \mathbf{B}^{\mathbf{H}} \overline{\mathbf{R}}_{2} \mathbf{B}\right.\right. \\
\left.\left.\cdot \mathbf{M M}^{\mathbf{H}} \mathbf{B}^{\mathbf{H}} \mathbf{B} \mathbf{M} \mathbf{B}^{-1} \mathbf{Q}_{h}\left(\mathbf{B}^{-\mathbf{H}}\right)\right)\right\} \\
\cdot \operatorname{vec}\left\{\mathbf{I}_{N_{M}} \otimes \mathbf{r}_{1}\right\} \\
=\mathbf{J}_{N_{M}, 2 N_{M}-1} \operatorname{vec}\left\{\mathbf{Q}_{h}^{H}\left(\mathbf{B}^{-\mathbf{H}}\right) \mathbf{B}^{-\mathbf{H}} \mathbf{M}^{\mathbf{H}} \mathbf{B}^{\mathbf{H}} \overline{\mathbf{R}}_{2} \mathbf{B} \mathbf{M}\right\} .
\end{gathered}
$$

As in [6], the operator vecb $(\cdot)$ is denoted as

$$
\begin{aligned}
\operatorname{vecb} & \left\{\mathbf{B} ; 2 N_{M}-1\right\} \\
\triangleq & \left.\triangleq \mathbf{B}_{11}^{T}, \mathbf{B}_{21}^{T}, \ldots, \mathbf{B}_{p 1}^{T}, \mathbf{B}_{12}^{T}, \ldots, \mathbf{B}_{p N_{M}}^{T}\right]^{T} \\
& \in \mathbb{C}^{\left(2 N_{M}-1\right) P N_{M},\left(2 N_{M}-1\right)}
\end{aligned}
$$

for any complex $\left(2 N_{M}-1\right) P \times\left(2 N_{M}-1\right) N_{M}$ block matrix $\mathbf{B}$,

$$
\mathbf{B}=\left[\begin{array}{cccc}
\mathbf{B}_{11} & \mathbf{B}_{12} & \cdots & \mathbf{B}_{1 N_{M}} \\
\mathbf{B}_{21} & \mathbf{B}_{22} & \cdots & \mathbf{B}_{2 N_{M}} \\
\vdots & \vdots & \vdots & \vdots \\
\mathbf{B}_{P 1} & \mathbf{B}_{P 2} & \cdots & \mathbf{B}_{P N_{M}}
\end{array}\right]
$$

where $\mathbf{B}_{i j} \in C^{\left(2 N_{M}-1\right),\left(2 N_{M}-1\right)}$ is the $i j$ th block. Equation (44) can be rewritten as

$$
\begin{aligned}
& \mathbf{J}_{N_{M}, 2 N_{M}-1} \operatorname{vecb}\{ \mathbf{Q}_{h}^{H}\left(\mathbf{B}^{-\mathbf{H}}\right) \mathbf{B}^{-\mathbf{H}} \mathbf{M}^{\mathbf{H}} \mathbf{B}^{\mathbf{H}} \overline{\mathbf{R}}_{2} \mathbf{B} \\
&\left.\cdot \mathbf{M M}^{\mathbf{H}} \mathbf{B}^{\mathbf{H}} \mathbf{B} \mathbf{M B}^{-1} \mathbf{Q}_{h}\left(\mathbf{B}^{-\mathbf{H}}\right), 2 N_{M}-1\right\} \mathbf{r}_{1} \\
&=\mathbf{J}_{N_{M}, 2 N_{M}-1} \operatorname{vec}\left\{\mathbf{Q}_{h}^{H}\left(\mathbf{B}^{-\mathbf{H}}\right) \mathbf{B}^{-\mathbf{H}} \mathbf{M}^{\mathbf{H}} \mathbf{B}^{\mathbf{H}} \overline{\mathbf{R}}_{2} \mathbf{B} \mathbf{M}\right\} .
\end{aligned}
$$

The optimum solution of the optimization problem (37) is given by

$$
\begin{aligned}
& \mathbf{r}_{1 \mathrm{opt}} \\
& =\left(\mathbf{J}_{N_{M}, 2 N_{M}-1}\right. \\
& \cdot \operatorname{vecb}\left\{\mathbf{Q}_{h}^{H}\left(\mathbf{B}^{-\mathbf{H}}\right) \mathbf{B}^{-\mathbf{H}} \mathbf{M}^{\mathbf{H}} \mathbf{B}^{\mathbf{H}} \overline{\mathbf{R}}_{2} \mathbf{B}\right. \\
& \left.\left.\cdot \mathbf{M M}^{\mathbf{H}} \mathbf{B}^{\mathbf{H}} \mathbf{B} \mathbf{M} \mathbf{B}^{-1} \mathbf{Q}_{h}\left(\mathbf{B}^{-\mathbf{H}}\right), 2 N_{M}-1\right\}\right)^{-1} \\
& \cdot \mathbf{J}_{N_{M}, 2 N_{M}-1} \operatorname{vec}\left\{\mathbf{Q}_{h}^{H}\left(\mathbf{B}^{-\mathbf{H}}\right) \mathbf{B}^{-\mathbf{H}} \mathbf{M}^{\mathbf{H}} \mathbf{B}^{\mathbf{H}} \overline{\mathbf{R}}_{2} \mathbf{B} \mathbf{M}\right\} .
\end{aligned}
$$

When $\mathbf{B}$ and $\mathbf{M}$ have different values, the results of the spatial spectrum estimation are different. The optimal selection of $\mathbf{B}$ and $\mathbf{M}$ could lead to the highest spatial spectrum amplitudes. Therefore, the global optimization problem is formed as

$$
\begin{aligned}
& {[\mathbf{B}, \mathbf{M}]} \\
& =\arg \max _{\mathbf{B}, \mathbf{M}} P_{\text {MUSIC }}\left(\mathbf{r}_{1 \text { opt }}\right) \\
& =\arg \max _{\mathbf{B}, \mathbf{M}} P_{\text {MUSIC }}\left(\left(\mathbf{J}_{N_{M}, 2 N_{M}-1}\right.\right. \\
& \cdot \operatorname{vecb}\left\{\mathbf{Q}_{h}^{H}\left(\mathbf{B}^{-\mathbf{H}}\right) \mathbf{B}^{-\mathbf{H}} \mathbf{M}^{\mathbf{H}} \mathbf{B}^{\mathbf{H}} \overline{\mathbf{R}}_{2} \mathbf{B}\right. \\
& \cdot \mathbf{M M}^{\mathbf{H}} \mathbf{B}^{\mathbf{H}} \mathbf{B} \mathbf{M B}^{-1} \mathbf{Q}_{h}\left(\mathbf{B}^{-\mathbf{H}}\right), \\
& \cdot \mathbf{J}_{N_{M}, 2 N_{M}-1} \\
& \left.\left.\cdot \operatorname{vec}\left\{\mathbf{Q}_{h}^{H}\left(\mathbf{B}^{-\mathbf{H}}\right) \mathbf{B}^{-\mathbf{H}} \mathbf{M}^{\mathbf{H}} \mathbf{B}^{\mathbf{H}} \overline{\mathbf{R}}_{2} \mathbf{B} \mathbf{M}\right\}\right)\right)^{-1} \\
& =\arg \min _{\mathbf{B}, \mathbf{M}} \frac{1}{P_{\text {MUSIC }}(f(\mathbf{B}, \mathbf{M}))},
\end{aligned}
$$

where $P_{\text {MUSIC }}(\cdot)$ is the MUSIC [20] spatial spectrum, which is constructed by $\mathbf{r}_{1 \mathrm{opt}}$ and

$$
\begin{aligned}
& f(\mathbf{B}, \mathbf{M}) \\
& =\left(\mathbf{J}_{N_{M}, 2 N_{M}-1}\right. \\
& \cdot \operatorname{vecb}\left\{\mathbf{Q}_{h}^{H}\left(\mathbf{B}^{-\mathbf{H}}\right) \mathbf{B}^{-\mathbf{H}} \mathbf{M}^{\mathbf{H}} \mathbf{B}^{\mathbf{H}} \overline{\mathbf{R}}_{2} \mathbf{B}\right. \\
& \left.\left.\cdot \mathbf{M} \mathbf{M}^{\mathbf{H}} \mathbf{B}^{\mathbf{H}} \mathbf{B} \mathbf{M} \mathbf{B}^{-1} \mathbf{Q}_{h}\left(\mathbf{B}^{-\mathbf{H}}\right), 2 N_{M}-1\right\}\right)^{-1} \\
& \cdot \mathbf{J}_{N_{M}, 2 N_{M}-1} \operatorname{vec}\left\{\mathbf{Q}_{h}^{H}\left(\mathbf{B}^{-\mathbf{H}}\right) \mathbf{B}^{-\mathbf{H}} \mathbf{M}^{\mathbf{H}} \mathbf{B}^{\mathbf{H}} \overline{\mathbf{R}}_{2} \mathbf{B} \mathbf{M}\right\} .
\end{aligned}
$$

For this nonconvex problem, we use the iterative alternating chain, which minimizes $1 / P\left(\mathbf{r}_{1 \text { opt }}\right)$ alternately over the two matrices $\mathbf{B}$ and $\mathbf{M}$. Suppose we have obtained the two matrices $\mathbf{B}^{(l)}$ and $\mathbf{M}^{(l)}$ at the $l$ th iteration. Then $\mathbf{B}^{(l+1)}$ and $\mathbf{M}^{(l+2)}$ can be solved by alternately optimizing the following two subproblems:

$$
\begin{gathered}
\mathbf{B}^{(l+1)}=\arg \min _{\mathbf{B}} \frac{1}{P\left(f\left(\mathbf{B}, \mathbf{M}^{(l)}\right)\right)}, \\
\mathbf{M}^{(l+2)}=\arg \min _{\mathbf{M}} \frac{1}{P\left(f\left(\mathbf{B}^{(l+1)}, \mathbf{M}\right)\right)} .
\end{gathered}
$$

Both (51) and (52) are convex. This observation motivates us to use the convex chain approach to solve the original nonconvex optimization problem in (49). We now consider the iterative alternating optimization routine

$$
\mathbf{B}^{(0)} \longrightarrow \mathbf{M}^{(1)} \longrightarrow \cdots \longrightarrow \mathbf{M}^{(2 l-1)} \longrightarrow \mathbf{B}^{(2 l)} \longrightarrow \cdots
$$

We can start from $\mathbf{B}^{(0)}$ and $\mathbf{M}^{(0)}$. Here, $\mathbf{B}^{(0)}$ and $\mathbf{M}^{(0)}$ are the initial vectors, and we define the covariance matrix with 
Initialize the virtual L-shaped uniform linear array with the same elements number as the real array, the distance of the adjacent elements as a random number between $0.01 \lambda$ to $0.5 \lambda$. We obtain the $\mathbf{B}^{(0)}$ with (20) and initialize $\mathbf{M}^{(0)}$ by the estimation method of the mutual coupling matrix in [6] without auxiliary elements.

For $l=0,1,2, \ldots$ do

(1) Compute the array data vector of the virtual array by (19).

(2) Compute the forward-backward smoothing covariance matrix by (23).

(3) Perform the local optimization for $\mathbf{B}^{(l+1)}$ by (51) and the local optimization for $\mathbf{M}^{(l+2)}$ by (52).

(4) If "strong" convergence is achieved by (55), the loop is break and the optimization of the spatial estimation is achieved end for by application of MUSIC algorithm with the covariance matrix obtained by (54). Otherwise, the loop continues.

Algorithm 1: CC-MUSIC.

the coupling of $\overline{\mathbf{R}}_{2}^{2 l}$ and $\overline{\mathbf{R}}_{2}^{2 l+1}$ of the $2 l$ th and $(2 l+1)$ th iterative routine as

$$
\begin{gathered}
\overline{\mathbf{R}}_{2}^{2 l}=\mathbf{B} \mathbf{M}^{(2 l-1)} \mathbf{R}_{1}\left(\mathbf{M}^{(2 l-1)}\right)^{\mathbf{H}} \mathbf{B}^{\mathbf{H}}, \\
\overline{\mathbf{R}}_{2}^{(2 l+1)}=\mathbf{B}^{(2 l)} \mathbf{M} \mathbf{R}_{1} \mathbf{M}^{\mathbf{H}}\left(\mathbf{B}^{(2 l)}\right)^{\mathbf{H}} .
\end{gathered}
$$

while, practically, "strong" convergence is achieved when

$$
\max \left(\begin{array}{c}
\left\|\mathbf{B}^{(2 l+2)}-\mathbf{B}^{(2 l)}\right\|_{F}, \\
\left\|\mathbf{M}^{(2 l+1)}-\mathbf{M}^{(2 l-1)}\right\|_{F},
\end{array}\right)<\delta \leq 1,
$$

where $\|\cdot\|_{F}$ is the Frobenius norm. An iterative procedure should be adopted, which is shown in Algorithm 1. In each iteration of the alternating optimization routine, a convex optimization is performed. Therefore we refer to this algorithm as Convex Chain MUSIC (CC-MUSIC).

Remark 1. It should be pointed out that the CC-MUSIC algorithm does not always converge to the real DOAs in the case of low SNR. Although its convergence value is not accurate, it is a simple and effective approach to achieve satisfactory subspace estimation. If it oscillates around a lower level value, this means that the objective function has attained a lower value. Then the algorithm is terminated and the subspace estimate can be obtained from the minimum point among all the iterations. The estimate is an approximation of the real DOAs. The number of the loops is related to the convergence coefficient $\delta$ in (55). If $\delta$ is much smaller, the convergence is "strong" and the number of the loops would be over thirty. To compromise between the convergence speed and the convergence accuracy, we could set a maximum of the number of the loops, such as 30. As the elements of the vectors $\boldsymbol{\rho}$ and $\hat{\boldsymbol{\rho}}^{(2)}$ are related to the distance of two antenna elements, we could define a linear factor $\gamma$ and we have $\mathbf{M}=\gamma \mathbf{M}^{(0)}$ for each search. Hence the number of the search parameter of $\mathbf{M}$ is reduced to one. Moreover, $\mathbf{B}$ is determined by one parameter which is the value of the adjacent distances of the elements of the virtual array. Hence the computational complexity of two searches in each loop is low.

\section{Simulation and Analysis}

In this section, we conduct several simulations to assess the proposed approach. We assume that the received array is an L-shaped nonuniform linear array with $N_{1} x$-axis and $N_{2} y$ axis receiving antenna elements, respectively. The antenna elements are of the equal gains and are spaced with the base distance of a half wavelength. We compare the performance of CC-MUSIC algorithm with three other approaches. They are the approaches which combine the MUSIC algorithm based on the spatial smoothing technique with the virtual array technique in the presence of the unknown coupling, the known coupling, and the estimated coupling coefficients by the estimation method in [6] without auxiliary elements. We consider targets with the same ranges and azimuths but with the different elevations.

In the first simulation, we set $N_{1}=N_{2}=40$. Both of the distance aggregates of the adjacent elements are $\begin{array}{lllllllllllllllllll}1 & 1 & 1 & 1\end{array}$ 12113111113111121111121112111311 $\left.2 \begin{array}{lll}1 & 1 & 1\end{array}\right\}$, where the " 1 " is a half wavelength, the " 2 " is two times of a half wavelength, and " 3 " is three times of a half wavelength. The vector $\bar{\rho}=[1,0.85+0.88 i, 0.59+0.56 i]^{T}$, and $p 1=3$. Moreover, $p 2=1$ and the vector $\widehat{\boldsymbol{\rho}}^{(2)}=[0.55-$ $0.16 i]^{T}$. We consider three targets with the different elevations which are $3^{\circ}, 6^{\circ}$, and $20^{\circ}$. The spatial spectrum of the first simulation with $5 \mathrm{~dB}$ SNR in each receiving antenna element is shown in Figure 2. It is shown that the proposed approach is effective for the estimation of the three targets. However, the three other approaches fail to resolve the targets. The spatial spectrum of the first simulation with $10 \mathrm{~dB}$ SNR in each receiving antenna element is shown in Figure 3. Although all of the four approaches are effective for the estimation of the three targets, the proposed approach achieves higher spatial amplitude than the other approaches.

We consider the estimation bias of the four approaches in the case of SNR $=0 \mathrm{~dB} \sim 30 \mathrm{~dB}$. Fifty simulations are carried on for each different SNR. The estimation bias in each Monte Carlo simulation is measured by the root-meansquared error (RMSE) as $\operatorname{RMSE}_{m}=\sqrt{\sum_{k=1}^{K}\left(\widehat{\varphi}_{m k}-\varphi_{k}\right)^{2} / K}$, where $\widehat{\varphi}_{m k}$ is the estimate of $\varphi_{k}$ in the $m$ th Monte Carlo simulation and $K$ is the number of the signals. The estimation bias is the mean of the fifty simulations bias, which is equal to $\sum_{m=1}^{M} \mathrm{RMSE}_{m} / M$, where $M$ is the repetition number of 


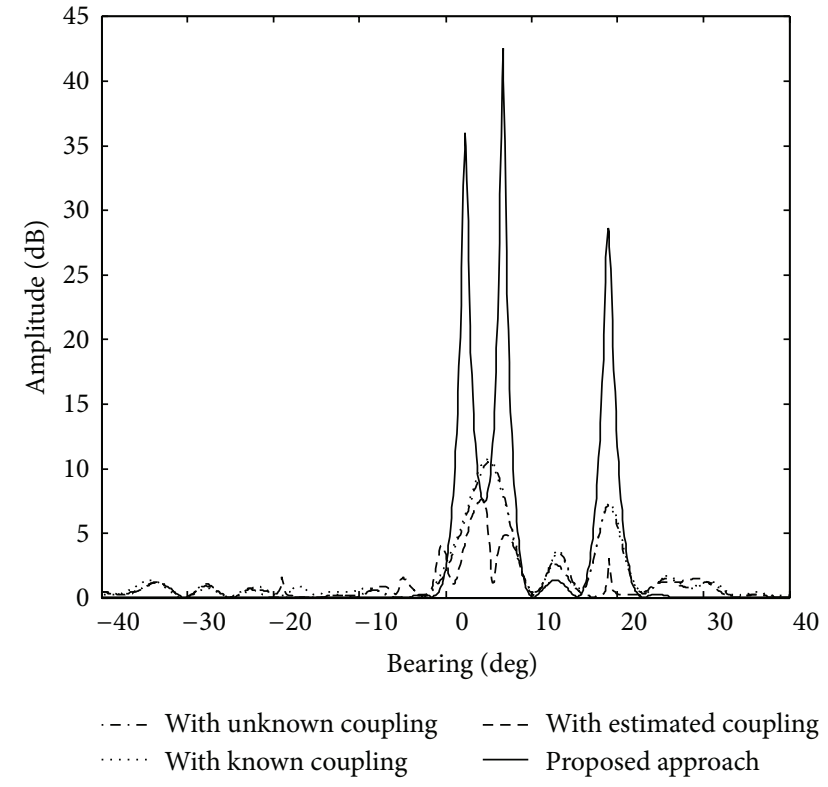

FIgURE 2: Spatial spectrum of the first simulation with $5 \mathrm{~dB}$ SNR.

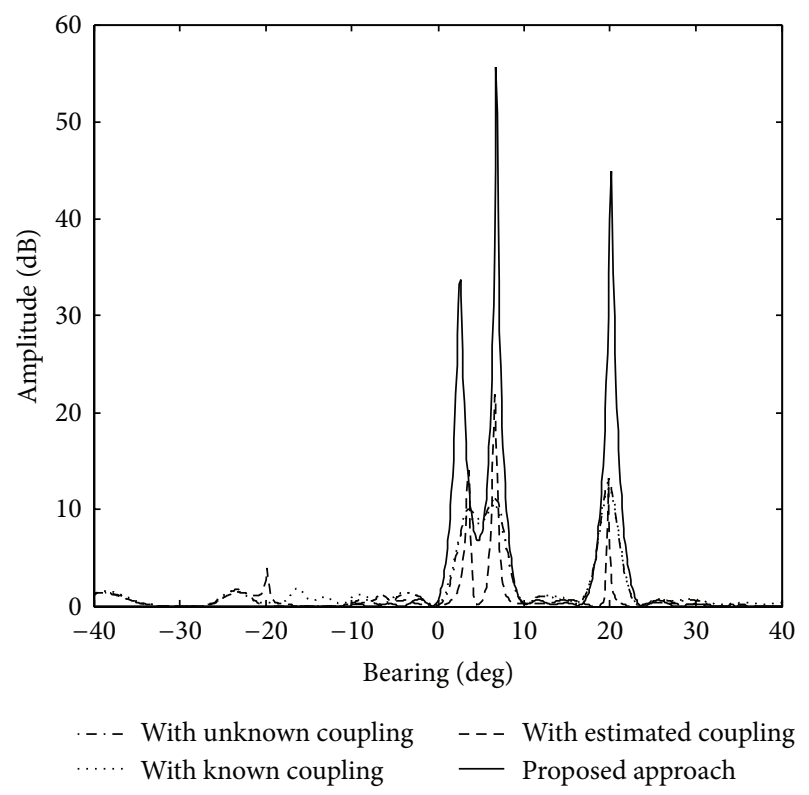

FIGURE 3: Spatial spectrum of the first simulation with $10 \mathrm{~dB}$ SNR.

the Monte Carlo simulation. If the approaches fail to resolve the three signals in some simulation repetitions, the $\mathrm{RMSE}_{m}$ of the repetitions are set to be $15^{\circ}$. The RMSE of the first simulation versus SNR is shown in Figure 4. It is shown that the proposed approach has the smallest RMSE. The variance of the first simulation versus SNR is also calculated. The result is shown in Figure 5. It is shown that the proposed approach has smallest variance.

The second simulation is carried out to evaluate the estimation bias and variance of the four approaches in different simulation conditions. Keep the other conditions of the array the same as the first simulation and consider the

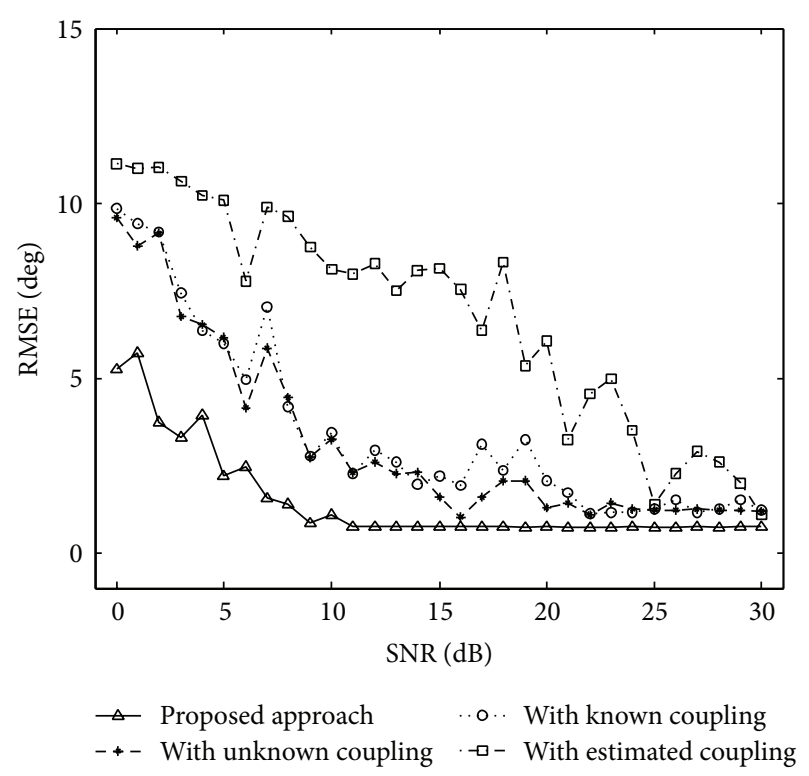

FIGURE 4: RMSE of the first simulation versus SNR.

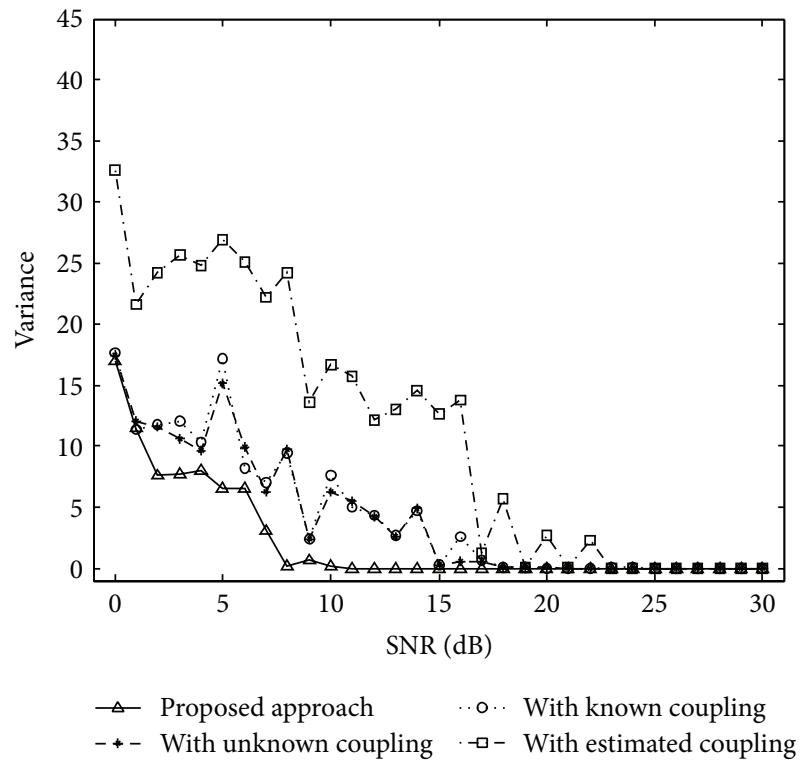

FIGURE 5: Variance of the first simulation versus SNR.

different vector $\bar{\rho}$ and the different target information. We consider $\overline{\boldsymbol{\rho}}=[1,0.85+0.88 i, 0.75+0.70 i, 0.61+0.6 i, 0.53+$ $0.50 i, 0.4+0.39 i, 0.2+0.1 i]^{T}$, and $p 1=7$. Two targets have the different elevations which are $3^{\circ}$ and $5^{\circ}$. The RMSE of the second simulation versus SNR is shown in Figure 6. It is shown that the proposed approach also has smallest RMSE. The variance of the second simulation versus SNR is shown in Figure 7. It is shown that the proposed approach has smallest variance.

In the third simulation, we keep the other conditions of the array the same as the second simulation and consider the different array antenna number and the different target elevations. The number of array antennas is 32 and two targets 


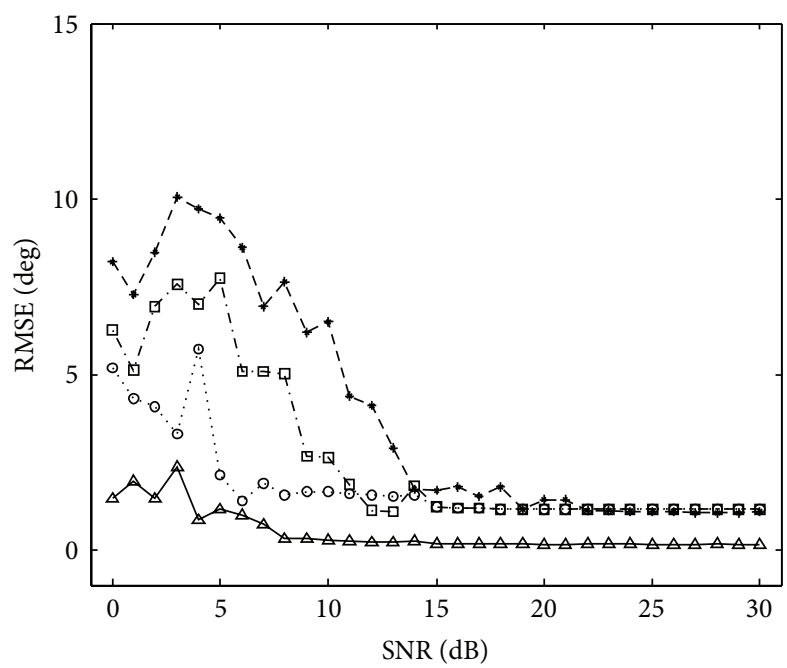

$\triangle$ Proposed approach $\quad$.... With known coupling

- - With unknown coupling - - - With estimated coupling

FIGURE 6: RMSE of the second simulation versus SNR.

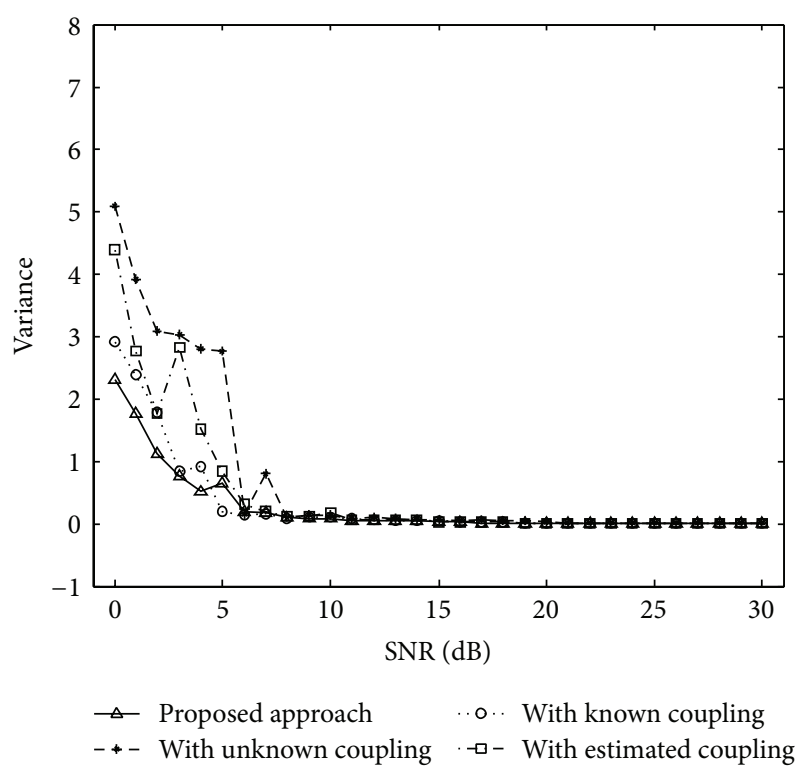

FIGURE 7: Variance of the second simulation versus SNR.

have the different elevations which are $-6^{\circ}$ and $-1^{\circ}$. The RMSE of the second simulation versus SNR is shown in Figure 8. It is shown that the proposed approach has the smallest RMSE. The variance of the second simulation versus SNR is shown in Figure 9. It is shown that the proposed approach has smallest variance.

\section{Conclusion}

A CC-MUISIC algorithm for the mutual coupling correction of the nonuniform L-shaped array with the single snapshot has been proposed. In applying the convex chain optimization, the computational complexity is reduced. Basically, the

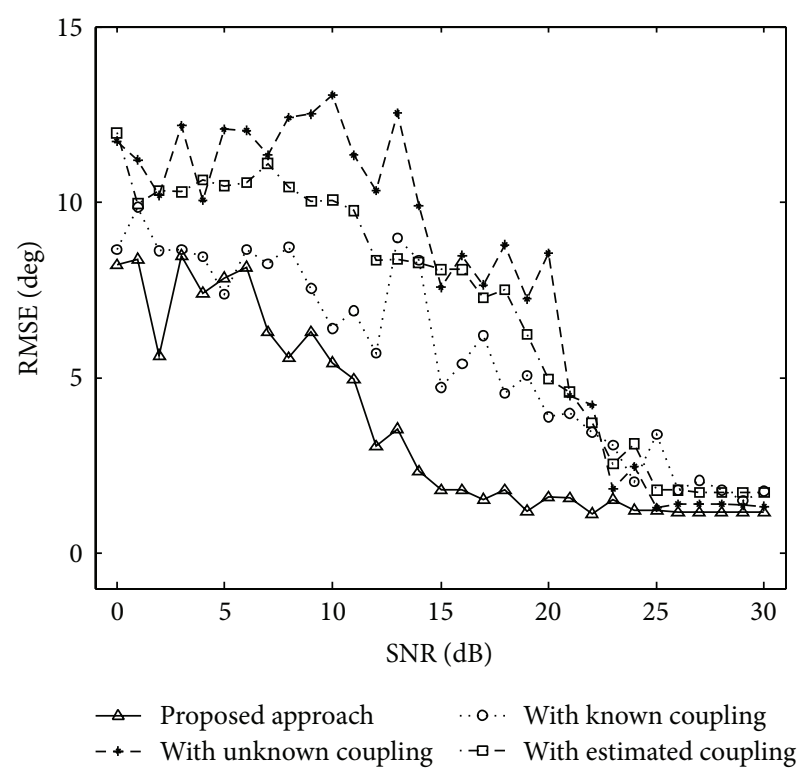

FIGURE 8: RMSE of the third simulation versus SNR.

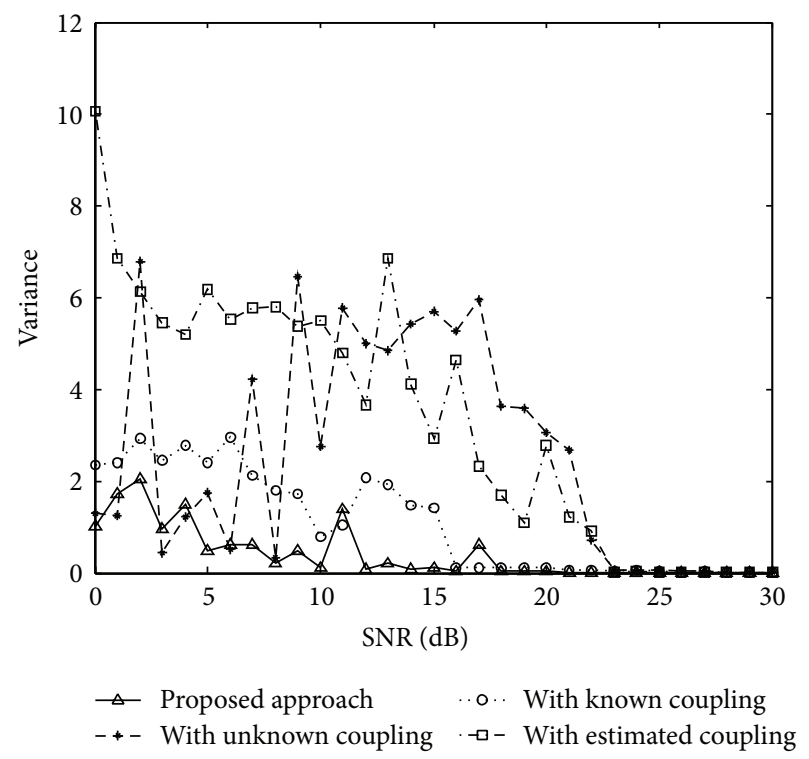

FIGURE 9: Variance of the third simulation versus SNR.

optimization routine is a process to find the optimization of the interpolated transformation matrix and the mutual coupling matrix for the spatial spectrum estimation of the multisources which are treated as the coherent signal sources for better resolution and estimation. We compared the performance of the CC-MUSIC algorithm with the approaches which combine the MUSIC algorithm based on the spatial smoothing technique with the virtual array technique in the presence of the unknown coupling, the known coupling, and the estimated coupling. The CC-MUSIC algorithm is more robust and more accurate. The simulation results demonstrate the validity of the proposed method in terms of the statistics of bias and variance. Although the proposed algorithm is for the L-shaped nonuniform linear array, it also 
suits other types of array, such as the circular array and the semicircular array.

\section{Conflict of Interests}

The authors declare that there is no conflict of interests regarding the publication of this paper.

\section{Acknowledgments}

The authors would like to thank the anonymous reviewers for their constructive comments, which helped improve the presentation of the paper. And this paper is partially supported by the National Natural Science Foundation of China (Grant no. 61301209).

\section{References}

[1] Y. Hua, T. K. Sarkar, and D. D. Weiner, "An L-shaped array for estimating 2-D directions of wave arrival," IEEE Transactions on Antennas and Propagation, vol. 39, no. 2, pp. 143-146, 1991.

[2] B. Friedlander and A. J. Weiss, "Direction finding in the presence of mutual coupling," IEEE Transactions on Antennas and Propagation, vol. 39, no. 3, pp. 273-284, 1991.

[3] B. C. Ng and C. M. Samson, "Sensor-array calibration using a maximum-likelihood approach," IEEE Transactions on Antennas and Propagation, vol. 44, no. 6, pp. 827-835, 1996.

[4] M. Lin and L. Yang, "Blind calibration and DOA estimation with uniform circular arrays in the presence of mutual coupling," IEEE Antennas and Wireless Propagation Letters, vol. 5, no. 1, pp. 315-318, 2006.

[5] A. Paulraj and T. Kailath, "Direction of arrival estimation by eigenstructure methods with unknown sensor gain and phase," in Proceedings of the IEEE International Conference on Acoustics, Speech, and Signal Processing (ICASSP '85), vol. 10, pp. 640-643, April 1985.

[6] Z. Ye and C. Liu, "On the resiliency of MUSIC direction finding against antenna sensor coupling," IEEE Transactions on Antennas and Propagation, vol. 56, no. 2, pp. 3741-3780, 2008.

[7] F. Sellone and A. Serra, "A novel online mutual coupling compensation algorithm for uniform and linear arrays," IEEE Transactions on Signal Processing, vol. 55, no. 2, pp. 560-573, 2007.

[8] Q. Bao, C. C. Ko, and W. Zhi, "DOA estimation under unknown mutual coupling and multipath," IEEE Transactions on Aerospace and Electronic Systems, vol. 41, no. 2, pp. 565-573, 2005.

[9] C. K. E. Lau, R. S. Adve, and T. K. Sarkar, "Minimum norm mutual coupling compensation with applications in direction of arrival estimation," IEEE Transactions on Antennas and Propagation, vol. 52, no. 8, pp. 2034-2041, 2004.

[10] C. Qi, Z. Chen, Y. Zhang, and Y. Wang, "DOA estimation and self-calibration algorithm for multiple subarrays in the presence of mutual coupling," IEE Proceedings: Radar, Sonar and Navigation, vol. 153, no. 4, pp. 333-337, 2006.

[11] S. Henault, S. K. Podilchak, S. M. Mikki, and Y. M. Antar, "A methodology for mutual coupling estimation and compensation in antennas," IEEE Transactions on Antennas and Propagation, vol. 61, no. 3, pp. 1119-1131, 2013.
[12] B. H. Wang, H. T. Hui, and M. S. Leong, "Decoupled 2D direction of arrival estimation using compact uniform circular arrays in the presence of elevation-dependent mutual coupling," IEEE Transactions on Antennas and Propagation, vol. 58, no. 3, pp. 747-755, 2010.

[13] C. H. Niow and H. T. Hui, "Improved noise modeling with mutual coupling in receiving antenna arrays for direction-ofarrival estimation," IEEE Transactions on Wireless Communications, vol. 11, no. 4, pp. 1616-1621, 2012.

[14] H.-S. Lui, H. T. Hui, and M. S. Leong, "A note on the mutualcoupling problems in transmitting and receiving antenna arrays," IEEE Antennas and Propagation Magazine, vol. 51, no. 5, pp. 171-176, 2009.

[15] B. Liao, Z.-G. Zhang, and S.-C. Chan, "DOA estimation and tracking of ULAs with mutual coupling," IEEE Transactions on Aerospace and Electronic Systems, vol. 48, no. 1, pp. 891-905, 2012.

[16] B. H. Wang and H. T. Hui, "Wideband mutual coupling compensation for receiving antenna arrays using the system identification method," IET Microwaves, Antennas and Propagation, vol. 5, no. 2, pp. 184-191, 2011.

[17] B. Friedlander and A. J. Weiss, "Direction finding using spatial smoothing with interpolated arrays," IEEE Transactions on Aerospace and Electronic Systems, vol. 28, no. 2, pp. 574-587, 1992.

[18] A. J. Weiss and B. Friedlander, "Performance analysis of spatial smoothing with interpolated arrays," IEEE Transactions on Signal Processing, vol. 41, no. 5, pp. 1881-1892, 1993.

[19] S. U. Pillai and B. H. Kwon, "Forward/backward spatial smoothing techniques for coherent signal identification," IEEE Transactions on Acoustics, Speech, and Signal Processing, vol. 37, no. 1, pp. $8-15,1989$.

[20] R. O. Schmidt, "Multiple emitter location and signal parameter estimation," IEEE Transactions on Antennas and Propagation, vol. 34, no. 3, pp. 276-280, 1986. 


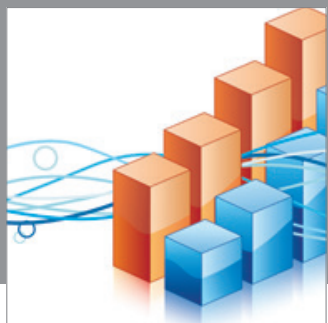

Advances in

Operations Research

mansans

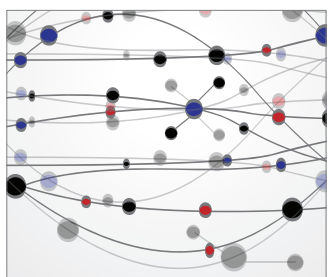

The Scientific World Journal
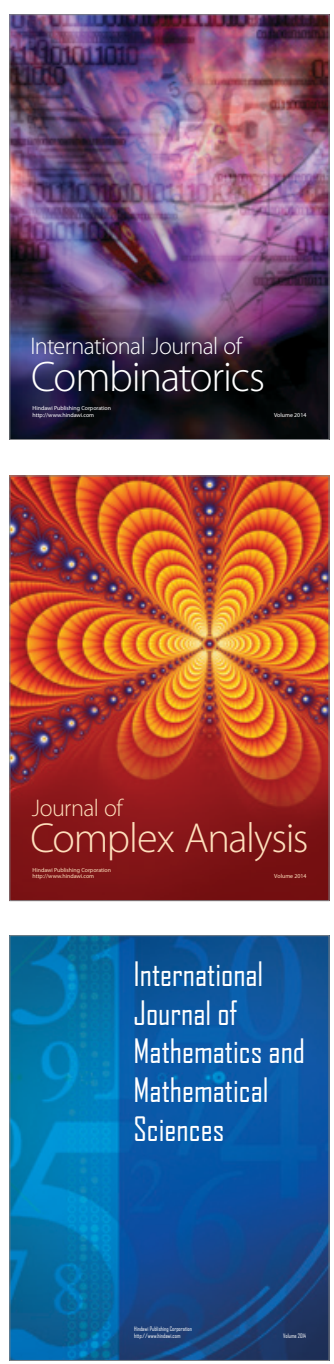
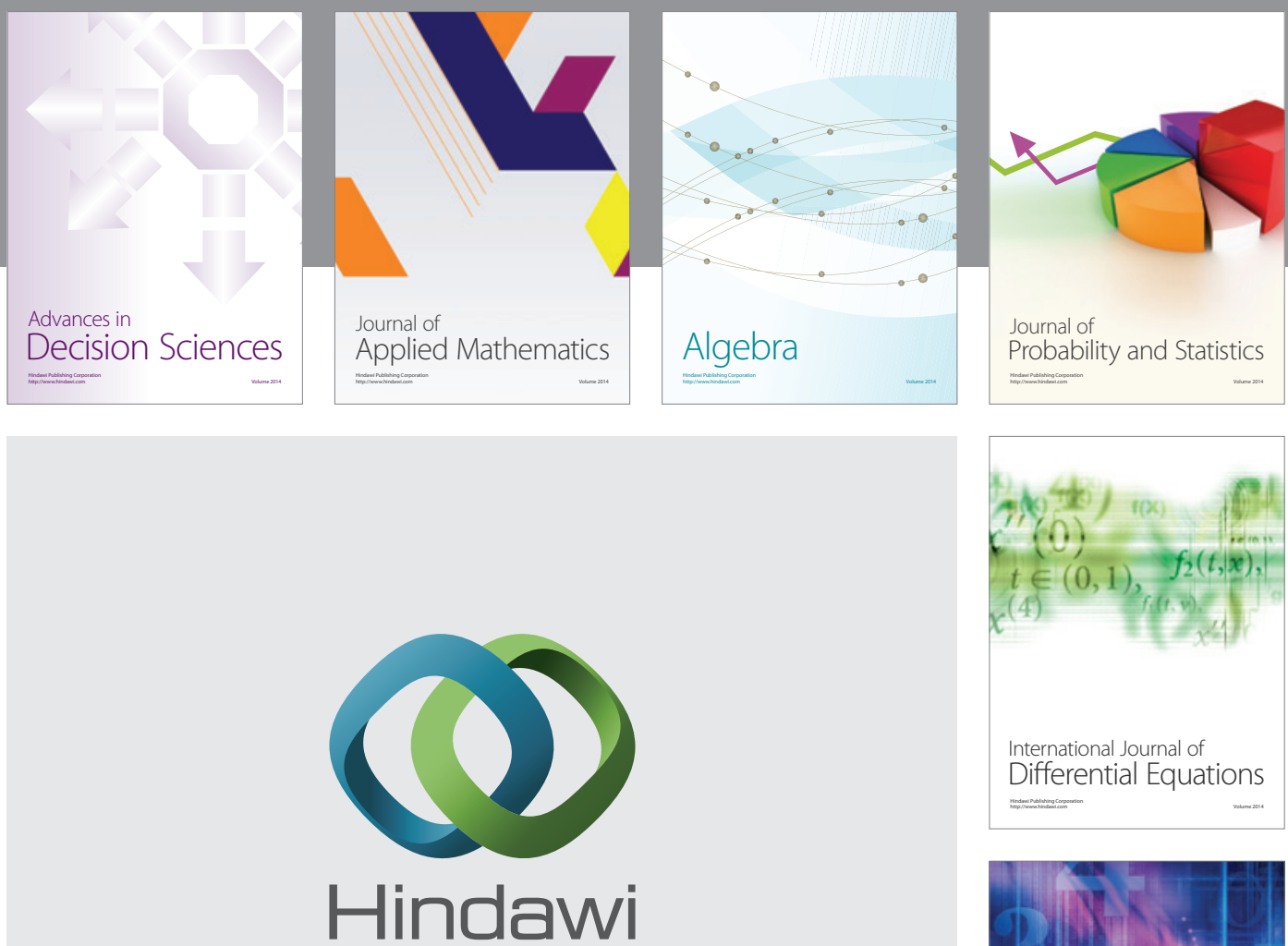

Submit your manuscripts at http://www.hindawi.com
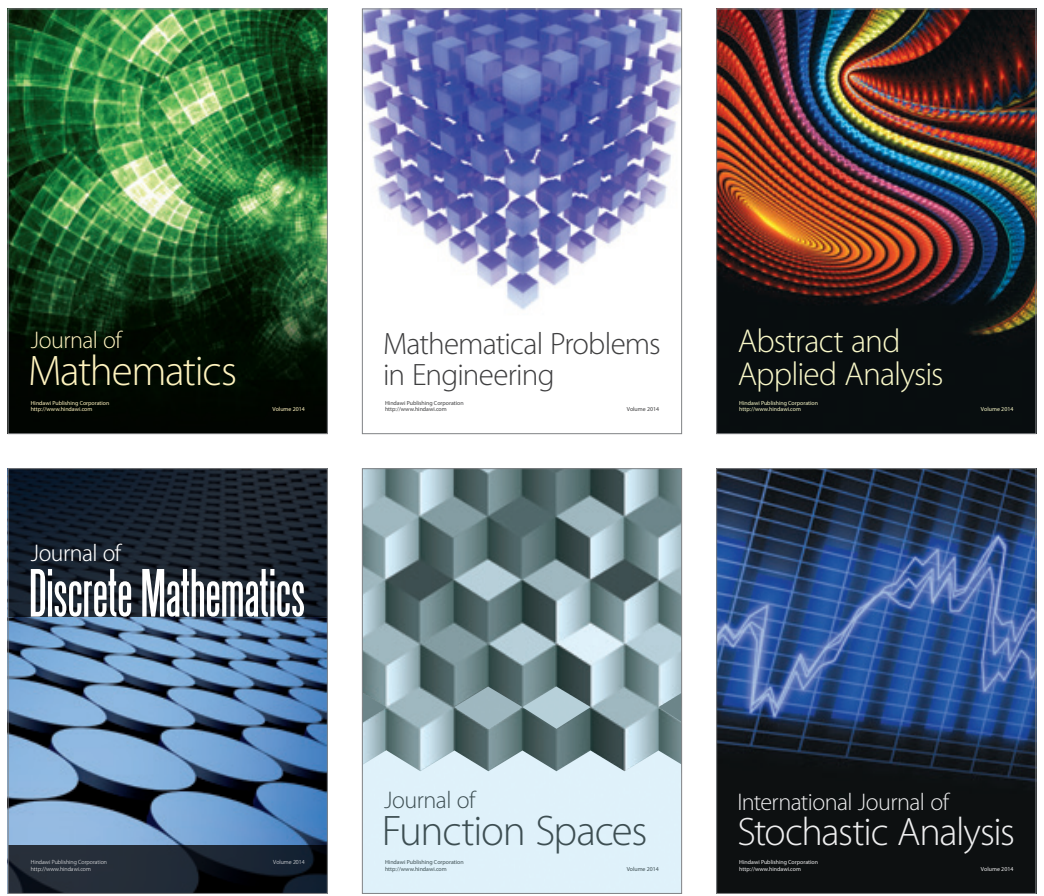

Journal of

Function Spaces

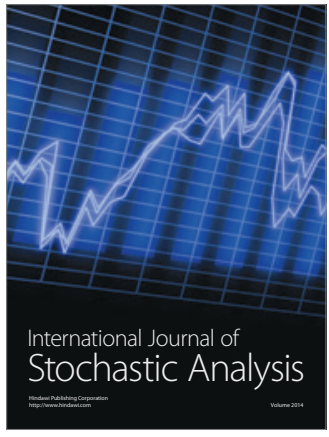

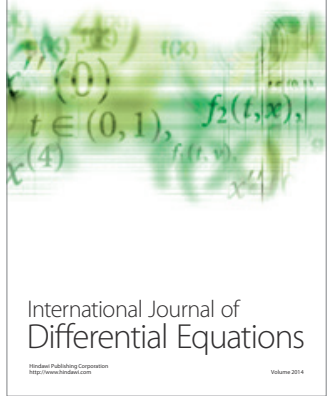
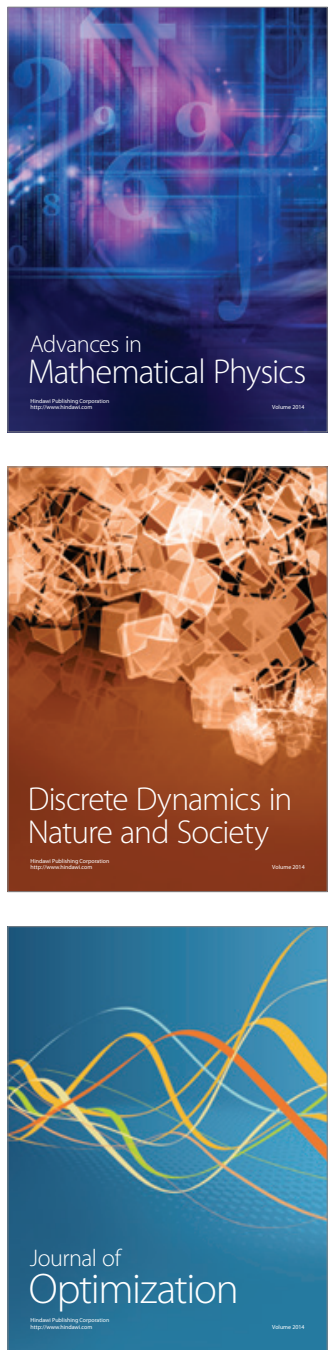